\title{
Study on the High-Birefringence Hollow-Core Anti-Resonant Fiber with Semicircular Cladding
}

\author{
Shuo Liu $\mathbb{D}^{1,2,3}$ Yuanwei Li, ${ }^{1,2}$ Rui Ma, ${ }^{1,2}$ Linwan Zhao, ${ }^{1,2}$ Jiaqi lv, ${ }^{1,2,3}$ and Xiaolong Dong ${ }^{1,2}$ \\ ${ }^{1}$ Center for Advanced Laser Technology, Hebei University of Technology, Tianjin 300401, China \\ ${ }^{2}$ Hebei Key Laboratory of Advanced Laser Technology and Equipment, Tianjin 300401, China \\ ${ }^{3}$ Tianjin Key Laboratory of Electronic Materials and Devices, Tianjin 300401, China
}

Correspondence should be addressed to Shuo Liu; liushuo@hebut.edu.cn

Received 12 February 2021; Accepted 2 May 2021; Published 22 May 2021

Academic Editor: Zhenao Bai

Copyright $(2021$ Shuo Liu et al. This is an open access article distributed under the Creative Commons Attribution License, which permits unrestricted use, distribution, and reproduction in any medium, provided the original work is properly cited.

\begin{abstract}
For the purpose of satisfying the demands of polarization-maintaining fibers for fiber optic gyroscopes, this article proposes a semicircular cladding birefringent hollow-core anti-resonant fiber. The influence of structural parameters on the birefringence, loss, and bending loss of the fiber is studied. The simulation results demonstrate that at $1550 \mathrm{~nm}$, the ultimate loss of the fundamental mode of $x$ and $y$ polarization is $1.76 \mathrm{~dB} / \mathrm{m}$ and $0.93 \mathrm{~dB} / \mathrm{m}$, respectively. The birefringence can reach $1 \times 10^{-4}$, and the wavelength range of birefringence greater than $10^{-4}$ can reach $60 \mathrm{~nm}$. This indicates that it has excellent bending properties. The proposed optical fiber has excellent performance in polarization maintenance and can supply ideas for the research of highprecision fiber optic gyroscopes and other optical devices.
\end{abstract}

\section{Introduction}

High-precision fiber optic gyroscopes have extremely important military and commercial application values $[1,2]$, and high-birefringence fiber as a core element has always been the focal point of scientific research. The sensitive ring is the core component of the fiber optic gyroscope made of polarization-maintaining fiber. The traditional polarization-maintaining fiber has a complicated manufacturing process, and $\mathrm{Ge}$ ion needs to be added to the fiber core. In the high-radiation environment, Ge ion will diffuse outwards which will result in a decrease in the refractive index of the fiber core, causing the loss of the fiber to increase greatly. Thus, the fiber optic gyroscope cannot work for a long time in the radiation environment [3]. Compared with traditional polarization-maintaining fibers, the microstructure fibers (MOFs) obtain birefringence through the asymmetry of structure. Therefore, it solves the problem of environmental adaptability under high-radiation working conditions from the source. Additionally, large bending loss and small birefringence are the serious shortcomings of traditional polarization-maintaining fibers, while polarization-maintaining MOFs can reduce bending loss and increase birefringence by changing the structure [4-6].

In recent years, MOFs have become a research hotspot due to their flexibility in design. According to the lightguiding mechanism, MOFs can be divided into hollow-core MOFs and solid-core MOFs. There are two kinds of lightguiding mechanisms of hollow-core MOFs: hollow-core photonic band gap fiber (HC-PBGF) and hollow-core antiresonant fiber (HC-ARF); light propagates by suppressing the coupling between core and cladding modes [7]. HC-ARF has the advantages of extremely low transmission loss, low bending loss, low dispersion, and large bandwidth which has aroused great interest [8-10]. These advantages make it have broad application prospects in pulse compression, ultrashort pulse transmission, data communication [11], supercontinuum generation [12], quantum optics, etc.

At present, the main issue of research is the influence of the core diameter of the hollow fiber on the total loss. Transmission loss decreases as the core diameter increases; however, the larger core will cause multimode operation. Therefore, careful engineering design of the geometry and 
size of the fiber is indispensable to achieve low loss in the single-mode working area [13]. For the purpose of adjusting the modal properties and reducing the total loss, several types of HC-ARF have been reported, including circular anti-resonant tubes $[14,15]$, ice cream cone-shaped negative curvature anti-resonant tubes [16], hexagonal structures [17], nested tubular structure [18], elliptical anti-resonant tube [19], and non-nested anti-resonant node [20]. Among them, the HC-ARF proposed by Mousavi et al. can simultaneously improve the birefringence and reduce the loss. The maximum birefringence of the HC-ARF is $1.5 \times 10^{-4}$, and the loss is less than $1 \mathrm{~dB} / \mathrm{m}[21]$.

In terms of polarization-maintaining fibers and optical devices, solid-core fibers can achieve high birefringence easily through asymmetric cladding structure. HC-ARF achieves high birefringence in a way different from solidcore fibers, and due to the light-guiding mechanism of HCARF, single-mode single-polarization can be achieved through mode coupling. In addition, HC-ARF has less nonlinearity, negligible dispersion, and low loss [22]. Compared with HC-PBGF, HC-ARF has an uncomplicated cladding structure, wider transmission passband, more flexible design freedom, and lower loss. Therefore, HC-ARF can be applied as a polarization-maintaining fiber to supply ideas for the research of high-precision fiber optic gyroscopes and other optical devices.

\section{Structure and Methods}

The cladding structure of HC-ARF is simple. In the communication band, it is generally composed of quartz ring and elliptical ring. The design structure of HC-ARF is shown in Figure 1. The cladding is a double semicircle ring structure. There are two asymmetric semicircles in the $x$ direction and the $y$ direction, and the arrangement is also different. In the $x$ direction, the first layer is the orange area near the core with thickness $t_{1}$, and the second layer is the purple area with thickness $t_{2}$. Thickness arrangement in the $y$ direction is opposite to that in the $x$ direction. $Z_{1}$ is the distance between two semicircles in the $x$ direction, and $Z_{2}$ is the distance between two semicircles in the $y$ direction. $R_{1}$ and $R_{2}$ are the radii of two semicircles in the $x$ direction, and $R_{3}$ and $R_{4}$ are the radii of two semicircles in the $y$ direction. $R_{a}$ and $R_{b}$ are the radii of the rectangular-like core in the $x$ and $y$ directions. Compared with double circle ring, double semicircle ring can change $Z_{1}$ in the same radian because the core of the semicircle can be the same one. And the change range of the parameters of the semicircle ring will be wider than the circle ring. The birefringence was caused by asymmetric semicircles in $x$ and $y$ directions. And the different arrangements of thickness in $x$ and $y$ directions will generate birefringence according to reference [21]. So, the structure can achieve high birefringence.

With the advancement of optical fiber manufacturing technology, especially the development of technology for hypocycloid-core Kagome fiber $[23,24]$ and negative curvature fiber $[25,26]$, it should be feasible to draw multilayer ARF. Similar hollow fibers have been made by stacking and stretching processes [27]. We only need to use different sizes

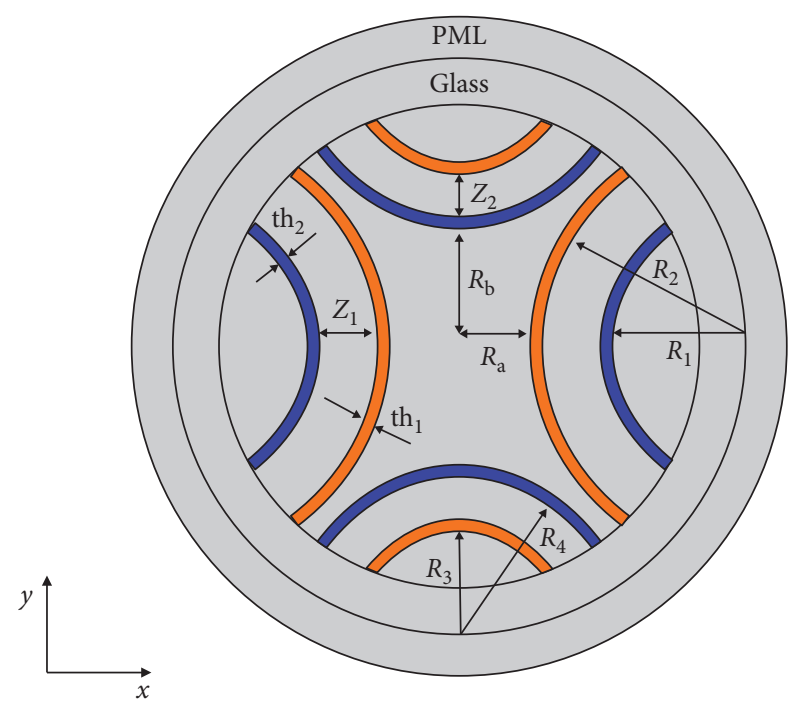

FIgURE 1: Structure of the proposed fiber.

of rods and capillaries for proper stacking design. During the fiber draw process, the tension is effectively controlled to improve the bending loss of the fiber.

In the numerical simulation, the perfectly matched layer is used as the outer boundary condition, as shown in Figure 1. Assume that the light incident on the interface between the perfectly matched layer and the optical fiber outer layer is completely absorbed and there is no reflection. Silica with a refractive index of 1.444 is used as the optical fiber material. The structure parameters are $\mathrm{th}_{1}=1.37 \mu \mathrm{m}$, $\mathrm{th}_{2}=1.18 \mu \mathrm{m}, R_{1}=13 \mu \mathrm{m}, R_{2}=20.1 \mu \mathrm{m}, R_{3}=11.5 \mu \mathrm{m}$, and $R_{4}=17 \mu \mathrm{m}$. The semirings are distributed in the center of the fiber tube wall.

The optical phase difference through the high refractive index area with or without reflection can be expressed by the following formula:

$$
\Delta \Phi=\Phi_{1}-\Phi_{0}=(2 m-1) \pi
$$

where $m$ is any positive integer. When the core mode and cladding mode are uncoupled or weakly coupled, the loss of the fiber is small, and the limiting loss of the fiber can be calculated:

$$
\text { Loss }=8.686 k_{0} \operatorname{Im}\left(n_{\text {eff }}\right) \text {, }
$$

where $k_{0}=2 \pi / \lambda$ and $\operatorname{Im}\left(n_{\text {eff }}\right)$ is the imaginary part of the effective refractive index of the desired mode. When the thickness is determined as $\mathrm{th}_{1}=1.37 \mu \mathrm{m}$ and $\mathrm{th}_{2}=1.18 \mu \mathrm{m}$, the fiber resonance wavelength can be determined to be $1427 \mathrm{~nm}$ and $1229 \mathrm{~nm}$ according to formula (1). A high loss area is formed near the resonant wavelength. As shown in Figure 2, the gray region is a resonant region formed by two resonant wavelengths. In the resonant region, the light loss is large and light cannot be transmitted. Figure 2 shows the limiting loss curve from $1000 \mathrm{~nm}$ to $1700 \mathrm{~nm}$, and the limiting loss is calculated by formula (2). 


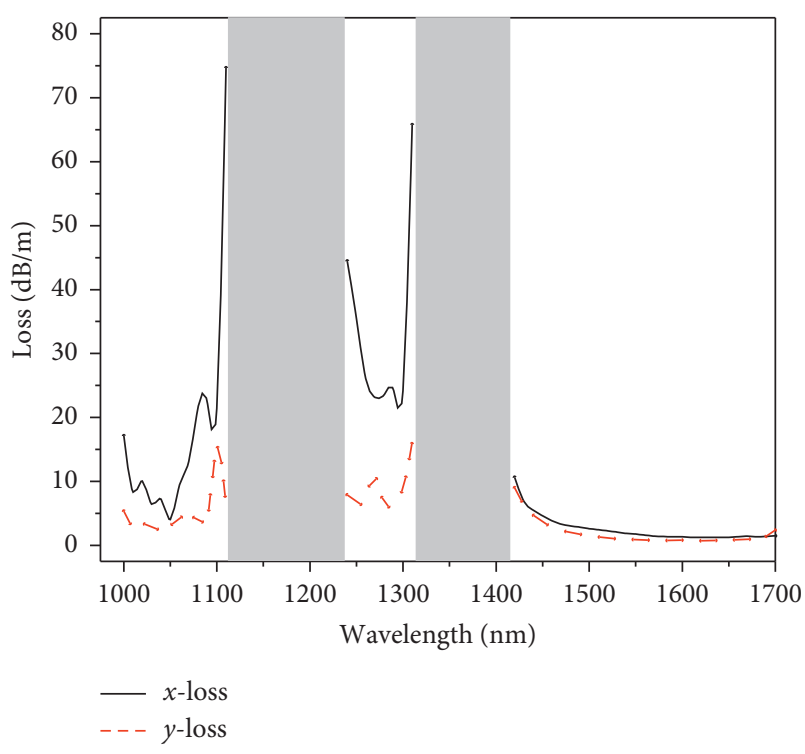

FIGURE 2: Loss of $x$ polarization mode and $y$ polarization mode as a function of wavelength.

As shown in Figures 3(a) and 3(b), in the first resonance region, when the light wavelength is $1.21 \mu \mathrm{m}$, the $x$-polarized fundamental mode has diffused into the cladding in the $y$ direction, and the $y$-polarized fundamental mode has been coupled with the cladding wall mode into the cladding. Therefore, the light leaks into the cladding in the $y$ direction in the first resonance region. As shown in Figures 3(c) and $3(\mathrm{~d})$, in the second resonance region, when the light wavelength is $1.42 \mu \mathrm{m}$, both the $x$ polarization fundamental mode and the $y$ polarization fundamental mode diffuse in the $x$ direction. Therefore, in the second resonance region, light diffuses from the $x$ direction to the cladding. As shown in Figures 3(e) and 3(f), when the wavelength of light is $1.55 \mu \mathrm{m}$, in the anti-resonant region, the light in either the $x$ direction or the $y$ direction is confined in the core.

As shown in Figure 3, the transmission of fundamental mode in optical fiber is actually the transmission of degenerate mode in two polarization directions. The refractive index of these two degenerate modes is extremely close and easy to couple. This phenomenon is not allowed in optical systems with polarization requirements. For the purpose of suppressing this phenomenon, increasing the propagation constant difference between the two lights is necessary. As shown in formula (3), increasing the propagation constant difference means increasing the mode birefringence of the fiber. The mode birefringence $B$ is defined as

$$
B=\frac{\left|\beta_{x}-\beta_{y}\right|}{k_{0}}=\left|n_{x}-n_{y}\right|
$$

where $\beta_{x}$ and $\beta_{y}$ are the propagation constants of $x$ and $y$ polarization modes, $k_{0}$ is the wave number, and $n_{x}$ and $n_{y}$ are the effective refractive indices of $x$ and $y$ polarization modes.

The birefringence in the anti-resonant region is shown in Figure 4. In the first anti-resonant region, the birefringence is small and does not satisfy the requirements. In the second anti-resonant region, the birefringence can reach $10^{-4}$. However, according to Figure 2, the loss in the second anti-resonant region is relatively large, which does not satisfy the requirements. In the third anti-resonant region, the birefringence first decreases, then increases, and then decreases. The birefringence is greater than $10^{-4}$ near the wavelength of $1550 \mathrm{~nm}$. According to Figure 2, in this region, the loss of the fundamental mode of the $x$ polarization and $y$ polarization of the fiber is very small. Therefore, the region of high birefringence and low loss appears near the wavelength of $1550 \mathrm{~nm}$.

\section{Results and Discussion}

The full-vector finite element method is used to explore the influence of the change of fiber structure parameters on the performance of the fiber. It mainly includes the effects on fiber birefringence, loss, and bending loss. The optimal structural parameters are obtained by changing some structural parameters. The discussion of structural parameters in this section selects a fixed wavelength of $1550 \mathrm{~nm}$.

3.1. The Influence of the Distance between Semicircular Rings. First of all, keep the other parameter values unchanged and change the spacing $Z_{1}$ of the double-layer semicircular ring in the $x$ direction, as shown in Figure 5; when the distance $Z_{1}$ between the semicircular rings increases, the birefringence increases, and the highest can reach $6 \times 10^{-5}$. The loss of the $x$ polarization fundamental mode first decreases and then increases, reaching a minimum of $0.86 \mathrm{~dB} / \mathrm{m}$ when $Z_{1}=5.73 \mu \mathrm{m}$. The loss of the $y$ polarization fundamental mode also decreases first and then increases. When $Z_{1}$ changes in the early period, the loss of the $y$ polarization fundamental mode is not obvious. When the distance of $Z_{1}$ increases, the loss of the $y$ polarization fundamental mode increases significantly. Compared with the loss of the fundamental mode of $x$ polarization, the loss of the fundamental mode of $y$ polarization has little change when $Z_{1}=4 \mu \mathrm{m}$ and $6 \mu \mathrm{m}$. When $Z_{1}=4.5 \mu \mathrm{m}$, the smallest loss of the $y$ polarization state is $0.72 \mathrm{~dB} / \mathrm{m}$. In the following analysis, $Z_{1}=5.73 \mu \mathrm{m}$ is selected as the optimal parameter; at this time, the losses of the $y$-polarized and $x$-polarized fundamental modes are $0.75 \mathrm{~dB} / \mathrm{m}$ and $0.86 \mathrm{~dB} / \mathrm{m}$, respectively, and the birefringence is $5.3 \times 10^{-5}$. Under this condition, although birefringence is not enough to satisfy the requirements of PMF, the loss of $x$ and $y$ polarization state is less than $1 \mathrm{~dB} / \mathrm{m}$.

It can be seen from Figure 5 that changing the distance $Z_{1}$ of the two semicircles in the $x$ direction has a great influence on the birefringence and $x$ polarization fundamental mode loss of the fiber, but compared with the loss of the $x$ polarization fundamental mode, the influence on the loss of the $y$ polarization fundamental mode is not obvious. This is because the distance Z2 of $y$-direction semicircle is fixed which only changes the distance $\mathrm{Z} 1$ of $x$-direction semicircle ring, resulting in the influence on $x$-polarization fundamental 


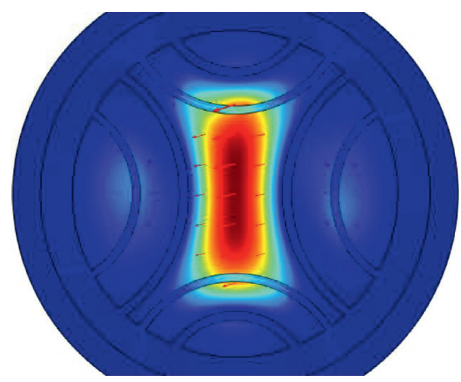

(a)

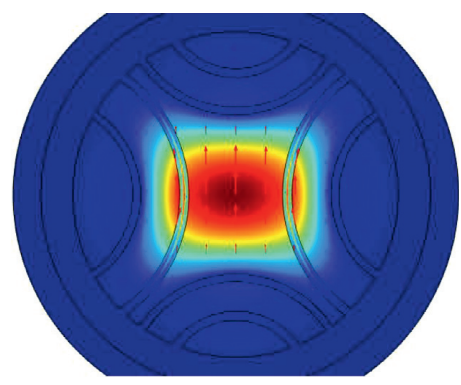

(d)

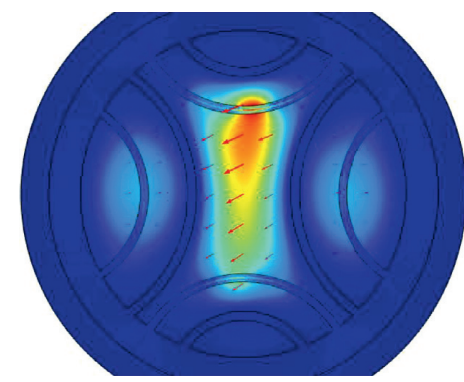

(b)

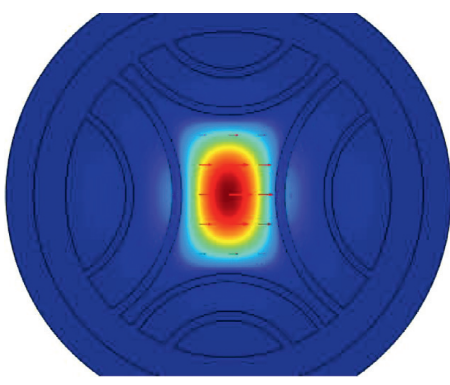

(e)

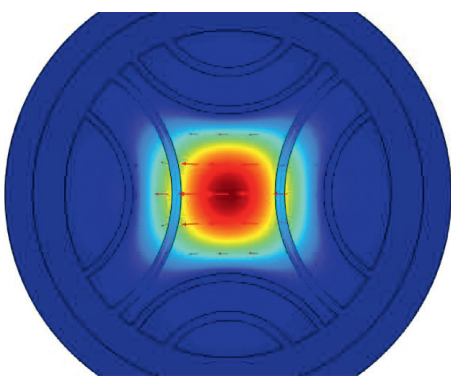

(c)

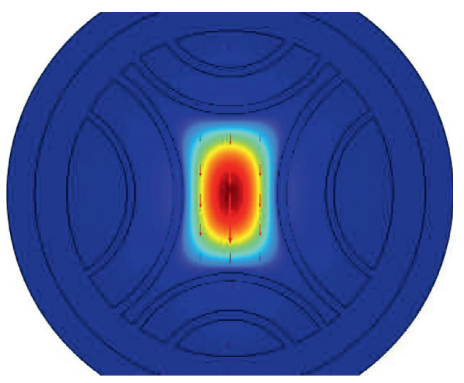

(f)

Figure 3: (a) $x$-polarized mode at $1.21 \mu \mathrm{m}$. (b) $y$-polarized mode at $1.21 \mu \mathrm{m}$. (c) $x$-polarized mode at $1.42 \mu \mathrm{m}$. (d) $y$-polarized mode at $1.42 \mu \mathrm{m}$. (e) $x$-polarized mode at $1.55 \mu \mathrm{m}$. (f) $y$-polarized mode at $1.55 \mu \mathrm{m}$.

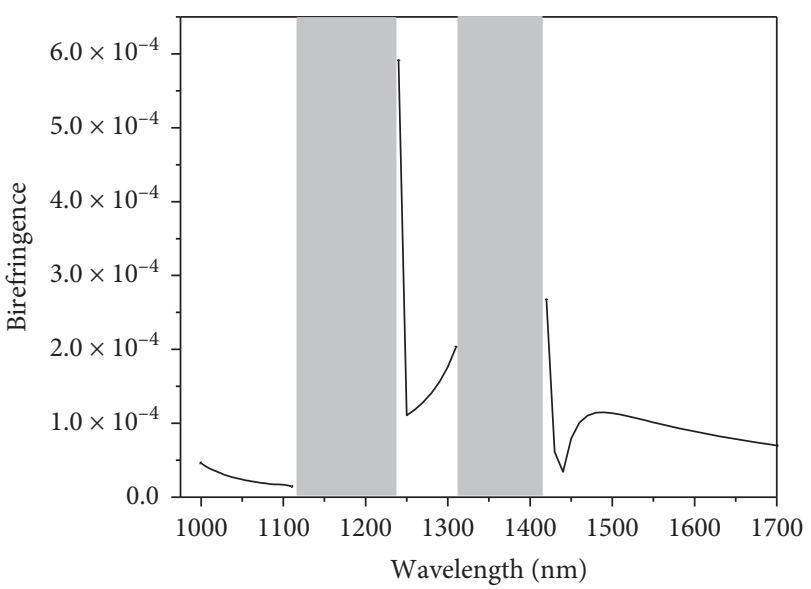

FIGURE 4: Birefringence as a function of wavelength.

mode inevitable more than the $y$-polarization fundamental mode. The rectangular core region is necessary to improve the birefringence. The radius of two semicircular rings in the $y$ direction is smaller than that of the two semicircular rings in the $x$ direction; as a result, the influence on the fundamental mode of $y$ polarization is relatively small. Therefore, in the following discussion on parameter changes, only the influence of the change of the structural parameters in the $x$ direction on the fundamental mode of the $x$ polarization is discussed and then the change of birefringence is discussed, so as to obtain the HC-ARF with high birefringence.

3.2. The Influence of the Size and Position of the Semicircle. Through the discussion of the distance $Z_{1}$ of the cladding semicircle in the $x$ direction in the previous section, it is determined that $Z_{1}=5.73 \mu \mathrm{m}$. This section mainly discusses the influence of the size and position of the double semicircle in the $x$ direction on the fundamental mode loss and birefringence. First, keep the position of the double-layer semicircle in the $x$ direction of the cladding unchanged, and then change the radius $R_{2}$ of the semicircle in the $x$ direction to obtain the optimal $R_{2}$ of the double-layer semicircle. Then, ensure that $R_{2}$ is unchanged and change the position of the center of the semicircle to obtain the optimal position of the double semicircle.

As shown in the schematic diagram of Figure 6(a), ensure that the center of the semicircle in the $x$ direction remains unchanged and then change the size of the semicircle by changing $R_{2}$. As shown in Figure 6(b), with the increase of $R_{2}$, the loss of the $x$ polarization fundamental mode gradually increases, and the loss of the $y$ polarization fundamental mode only increases slightly. However, the increase is small compared to the $x$ polarization fundamental mode loss. The birefringence between the $x$ and $y$ polarization fundamental modes increases significantly with the increase of $R_{2}$. When $R_{2}=21.8 \mu \mathrm{m}$, the $x$ and $y$ polarization fundamental mode losses are $1.76 \mathrm{~dB} / \mathrm{m}$ and $0.93 \mathrm{~dB} / \mathrm{m}$, respectively, and the birefringence is $1.0 \times 10^{-4}$. At this time, the loss is lower and the birefringence can also satisfy the requirements. When $R_{2}=22.8 \mu \mathrm{m}$, the maximum birefringence can reach $1.6 \times 10^{-4}$, but the losses in the basic modes of the $x$ and $y$ polarization are increased to $3.98 \mathrm{~dB} / \mathrm{m}$ and $1.31 \mathrm{~dB} / \mathrm{m}$, respectively. This shows that in pursuit of maximum birefringence, partial loss of the fundamental mode must be sacrificed.

As shown in Figure $7(\mathrm{a})$, keep the radius $R_{2}$ of the semicircular ring unchanged, change the position of the center of the semicircle, and define the distance from the 


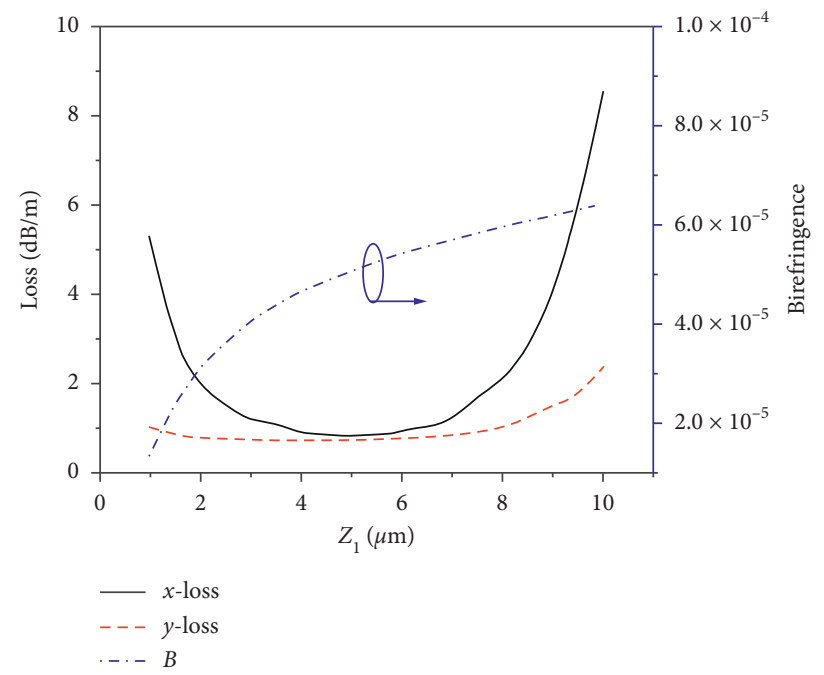

FIgURE 5: The loss of $x$ and $y$ polarization modes and birefringence as a function of $Z_{1}$.

center of the semicircle to the core as $Z_{c}$. As shown in Figure $7(\mathrm{~b})$, when $Z_{c}$ increases, the fundamental mode loss of $x$ polarization decreases significantly, the fundamental mode loss of $y$ polarization also decreases slightly but basically remains unchanged, and birefringence shows a significant reduction trend. When $Z_{c}=28.7 \mu \mathrm{m}$, the center of the circle is just right on the outer tube wall of the optical fiber, the $x$ and $y$ polarization fundamental mode losses are $1.76 \mathrm{~dB} / \mathrm{m}$ and $0.93 \mathrm{~dB} / \mathrm{m}$, respectively, and the birefringence is $1.0 \times 10^{-4}$. At this time, the loss of the optical fiber is low and the birefringence is high, which can satisfy the requirements.

Actually, both the increase of $R_{2}$ and the increase of $Z_{c}$ change the shape of the core region. Comparing Figures $6(\mathrm{~b})$ and 7(b), it can be seen that when $R_{2}$ increases or $Z_{c}$ decreases, the $x$ direction area of the core decreases. This in turn leads to an increase in birefringence, a significant increase in the loss of the fundamental mode of $x$ polarization, and a slight increase in the loss of the fundamental mode of $y$ polarization. The difference between these two changes is that the curvature in the $x$ direction of the core is different, but from the results, this difference cannot be reflected in Figures 6(b) and 7(b). Therefore, in the next section, we will only consider the influence of curvature change of semicircle ring in $x$ direction on fiber performance.

3.3. The Effect of Ellipticity. In this section, we study the change of the ellipticity (e) of the semicircular ring in the $x$ direction when the other parameters remain unchanged. The ellipticity can be defined as

$$
e=\frac{r_{a}}{r_{b}}
$$

As shown in Figure 8(a), $r_{a}$ and $r_{b}$, respectively, are the semimajor axis and semiminor axis of the elliptical semicircular ring of the cladding. When the other parameters remain unchanged, the ellipticity $e$ can be changed by changing $r_{b}$. As shown in Figure 8(b), when the ellipticity $e$ increases, the birefringence of the fiber decreases rapidly, indicating that the change of ellipticity $e$ will greatly influence the birefringence. At the same time, it can be seen from Figure 7(b) that when the ellipticity $e$ increases in the $x$ direction, the loss of the $x$-polarized fundamental mode first decreases and then increases. When $e=1.6$, the lowest loss obtained for the fundamental mode of $x$ polarization is $0.67 \mathrm{~dB} / \mathrm{m}$. The ellipticity $e$ also has the great influence on the $y$ polarization fundamental mode loss. As $e$ increases, the $y$ polarization fundamental mode loss keeps growing. This illustrates that when the ellipticity $e$ increases, the constraint on the $y$ polarization fundamental mode decreases, resulting in an increase in the $y$ polarization fundamental mode loss. And the birefringence decreases as the ellipticity increases. When $e=0.9$, the fundamental mode loss for the $x$ polarization is $1.87 \mathrm{~dB} / \mathrm{m}$ and that for the $y$ polarization is $0.88 \mathrm{~dB} /$ $\mathrm{m}$, and the birefringence is $1.05 \times 10^{-4}$. When $e=1$, the fundamental mode losses of the $x$ and $y$ polarizations are $1.76 \mathrm{~dB} / \mathrm{m}$ and $0.93 \mathrm{~dB} / \mathrm{m}$, respectively, and the birefringence is $1.0 \times 10^{-4}$. Compared with the result of $e=0.9$, when $e=1$, the loss of the $y$-polarized fundamental mode is higher, but the loss of the $x$-polarized fundamental mode is reduced more. Therefore, it can be obviously seen that when $r_{a}$ is unchanged, increasing $r_{b}$ appropriately can increase the birefringence and reduce the loss of the $y$ polarization fundamental mode, so that the fiber has better polarizationmaintaining performance. However, considering that the manufacturing process of the elliptical tube is more difficult, $e=1$ is selected in the following analysis.

3.4. Fiber Bending. In the fiber optic gyroscope, the polarization-maintaining fiber can be applied as the sensitive ring, and the sensitive ring is the core component of the fiber optic gyroscope. The better the bending performance of the designed fiber is, the smaller the volume of the fiber optic gyroscope can be and the more fields it can be applied to. Therefore, it is essential to analyze the bending loss of $\mathrm{HC}$ ARF. 

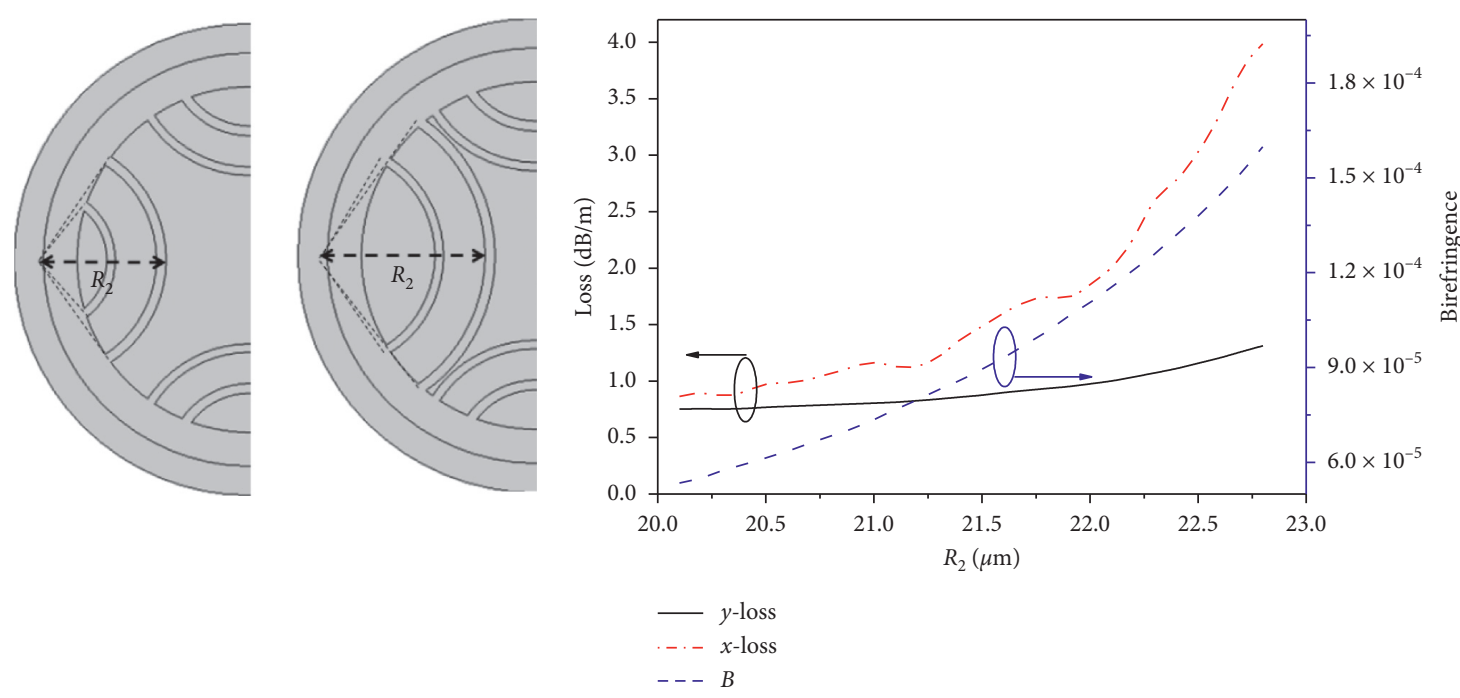

(a)

(b)

Figure 6: (a) The center of the circle is fixed and $R_{2}$ is changed. (b) $x$ and $y$ polarization mode loss and birefringence as a function of $R_{2}$.

The equivalent refractive index method is generally used to analyze the bending loss of the fiber. When the optical fiber is bent, the refractive index distribution of the optical fiber changes according to the change of the bending direction, the bending radius, and the angle. Therefore, when an optical fiber is bent, it can be considered that the refractive index of the ordinary optical fiber changes, and it only requires to calculate the refractive index of the bent fiber for numerical analysis. The equivalent refractive index model can be expressed as

$$
n_{\mathrm{eq}}(x, y)=n(x, y) \sqrt{1+\frac{2(x \cos \theta+y \sin \theta)}{R_{b}}},
$$

where $n_{\mathrm{eq}}(x, y)$ is the equivalent refractive index of the bent fiber, $n(x, y)$ is the refractive index of the straight fiber, $R_{b}$ is the bending radius of the fiber, and $\theta$ is the angle formed by the bending direction of the fiber and the $x$ axis. Because the designed fiber is asymmetric in both the $x$ direction and $y$ direction, different bending directions, as shown in Figure 9(a), will also affect the bending performance of the fiber. As shown in Figure 9, the intensity distribution of the fundamental mode is related to the bending direction of the fiber. So, the bending performance of the fiber is not quite the same.

As shown in Figure 10(a), when the fiber is bent in the $x$ direction, the wavelength is $1550 \mathrm{~nm}$, the bending radius is $10 \mathrm{~cm}$ and $5 \mathrm{~cm}$, the loss of the fundamental mode of the $x$ polarization has a small change, and the loss is $1.81 \mathrm{~dB} / \mathrm{m}$ and $1.96 \mathrm{~dB} / \mathrm{m}$, respectively, which is almost the same as $1.76 \mathrm{~dB} / \mathrm{m}$ of straight fiber. However, when the bending radius continues to decrease to $2 \mathrm{~cm}$, the $x$ polarization fundamental mode loss will suddenly increase to $3.32 \mathrm{~dB} / \mathrm{m}$, an increase of $1.56 \mathrm{~dB} / \mathrm{m}$. In Figure $10(\mathrm{~b})$, when the wavelength is $1550 \mathrm{~nm}$ and the bending radius is $10 \mathrm{~cm}$ and $5 \mathrm{~cm}$, the fundamental mode loss of the $y$ polarization and the loss of the straight fiber are $0.934 \mathrm{~dB} / \mathrm{m}, 0.938 \mathrm{~dB} / \mathrm{m}$, and $0.948 \mathrm{~dB} / \mathrm{m}$, respectively, showing a very small growth trend. But when the bending radius is reduced to $2 \mathrm{~cm}$, the $y$ polarization fundamental mode loss increases to $1.03 \mathrm{~dB} / \mathrm{m}$. When the fiber is bent in the $x$ direction and the bending radius is greater than $5 \mathrm{~cm}$, the bending loss of the optical fiber is relatively small and less than $1 \mathrm{~dB} / \mathrm{m}$, indicating that the optical fiber has superior bending performance when bending in the $x$ direction.

As shown in Figure 11(a), when the wavelength is $1550 \mathrm{~nm}$, the fiber is bent in the $y$ direction, the bending radius is $10 \mathrm{~cm}, 5 \mathrm{~cm}$, and $2 \mathrm{~cm}$, the $x$-polarized fundamental mode loss and the straight fiber loss hardly change, and the loss is $1.76 \mathrm{~dB} / \mathrm{m}, 1.76 \mathrm{~dB} / \mathrm{m}, 1.76 \mathrm{~dB} / \mathrm{m}$, and $1.77 \mathrm{~dB} / \mathrm{m}$, respectively, which is almost unaffected by fiber bending. In Figure 11(b), when the bending radius is $10 \mathrm{~cm}$ and $5 \mathrm{~cm}$, the $y$ polarization fundamental mode loss and the straight fiber loss change very little, and they are $0.93 \mathrm{~dB} / \mathrm{m}, 0.95 \mathrm{~dB} / \mathrm{m}$, and $1.02 \mathrm{~dB} / \mathrm{m}$. But when the bending radius is reduced to $2 \mathrm{~cm}$, the $y$ polarization fundamental mode loss increases significantly to $1.51 \mathrm{~dB} / \mathrm{m}$. It can be found that when the fiber is bent in the $y$ direction and the bending radius is greater than $5 \mathrm{~cm}$, the bending loss of the optical fiber is relatively small, indicating that the optical fiber has superior bending performance when bending in the $y$ direction.

Comparing Figures 10(a) and 11(a), it can be found that when the fiber is bent in the $x$ direction, it greatly influences the bending loss of the $x$ polarization fundamental mode. But when the fiber is bent in the $y$ direction, the fundamental mode bending loss of the $x$ polarization is basically unchanged. Comparing Figures 10(b) and 11(b), it can be found that when bending in the $x$ direction, with the change in the bending radius, the bending loss of the $y$ polarization fundamental mode changes less than the bending loss when bending in the $y$ direction. Therefore, if the optical fiber is made into a sensitive ring, when the light in the sensitive ring is $x$-polarized light and the fiber is bent in the $y$ direction, the 

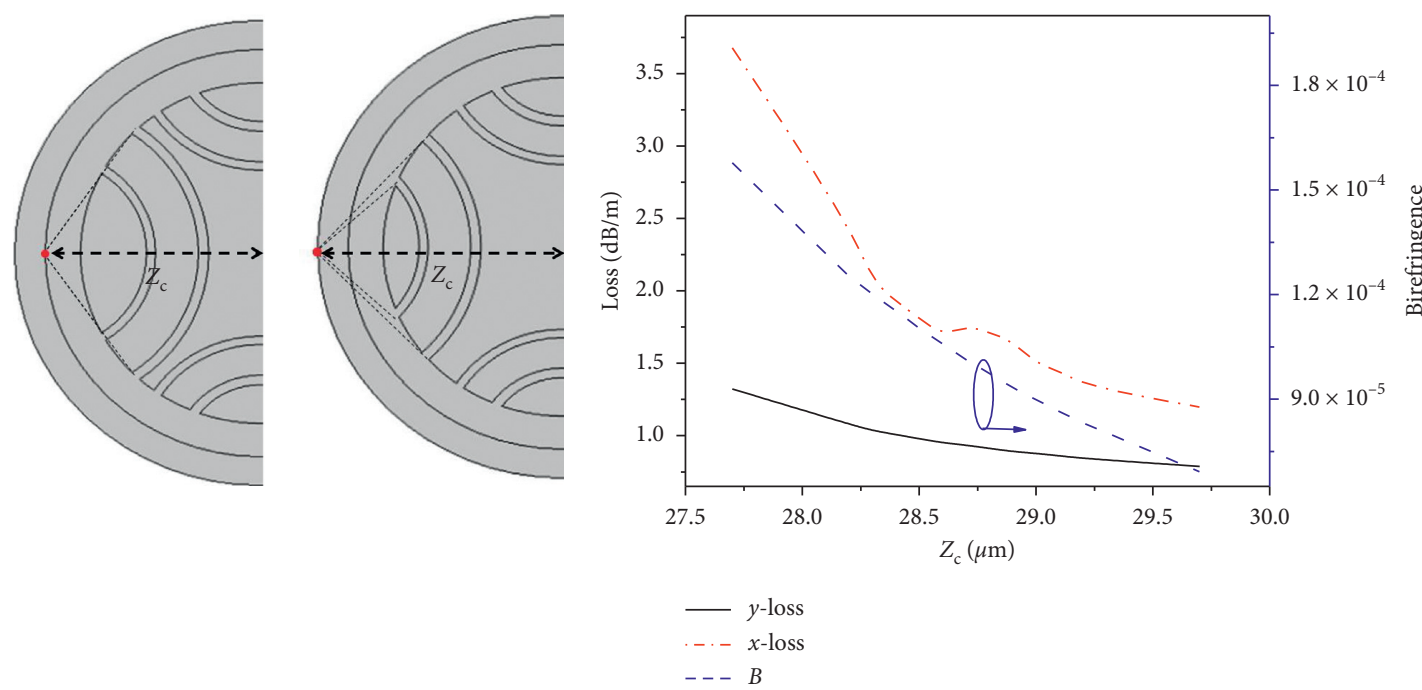

(a)

(b)

Figure 7: (a) The change of $Z_{c}$ with a fixed radius; (b) fixing radius, $x$ and $y$ polarization mode loss and birefringence as a function of $Z_{c}$.
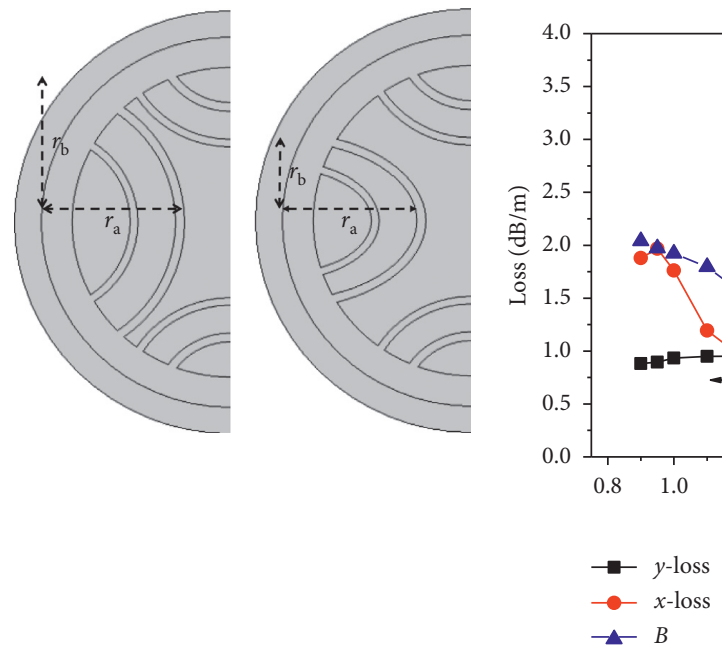

(a)

(b)

Figure 8: (a) Diagram of $e$ change. (b) $x$ and $y$ polarization mode loss and birefringence as a function of $e$.

loss is smaller. When the sensitive ring is $y$-polarized light and the fiber is bent in the $x$ direction, the bending loss will be smaller.

Fiber bending will also affect the birefringence. As shown in Figure 12(a), when the fiber is bent in the $x$ direction, the birefringence of the fiber will increase as the bending radius decreases. However, as shown in Figure 12(b), when the fiber is bent in the $y$ direction, the birefringence of the fiber tends to decrease, but the change is relatively small. When the bending radius is $5 \mathrm{~cm}$, the birefringence is still greater than $1.0 \times 10^{-4}$. This means that when the bending loss is within a reasonable range, the change of birefringence caused by bending is relatively small, which suffices the index requirements. 

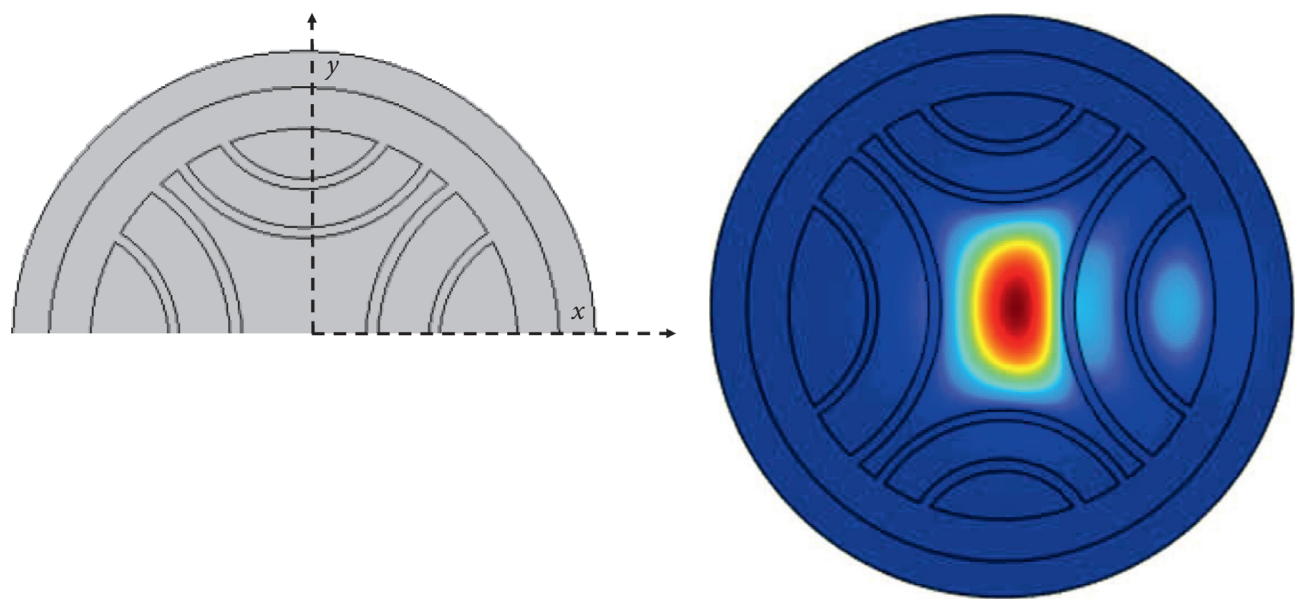

(a)

(b)

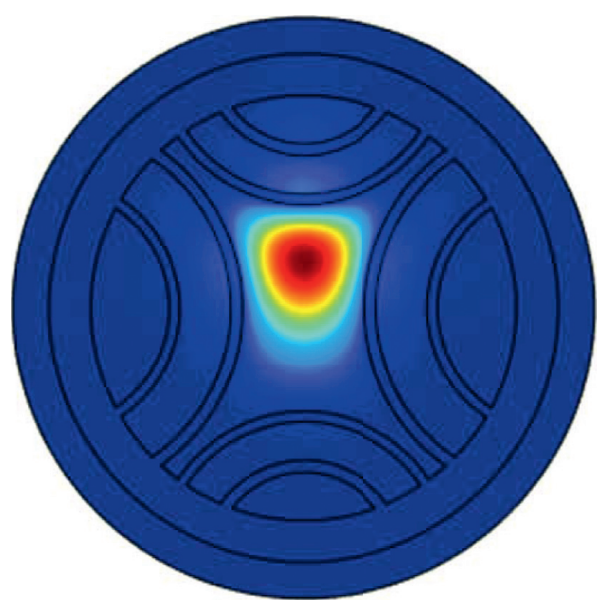

(c)

FIGURE 9: (a) Bending directions, fundamental mode in the wavelength of $1550 \mathrm{~nm}$ at (b) $x$ and (c) $y$ bending direction.

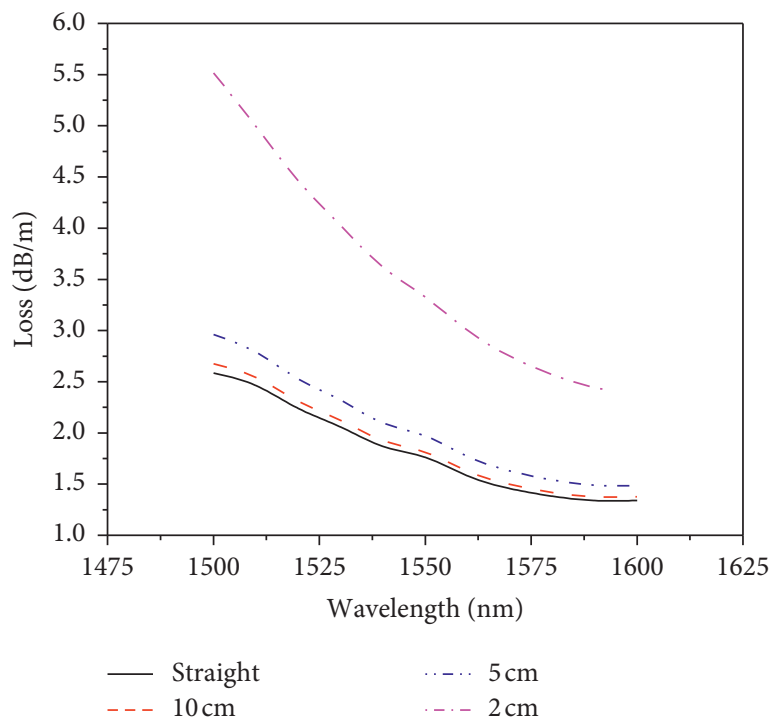

(a)

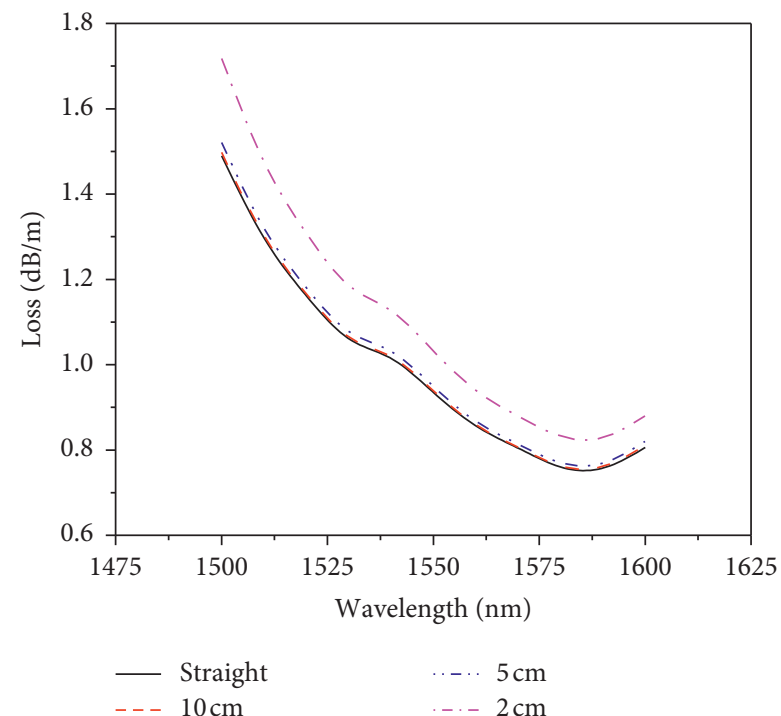

(b)

FiguRE 10: Fiber bends in $x$ direction, the bending loss of (a) $x$ polarization mode and (b) $y$ polarization mode curve at different bending radius. 


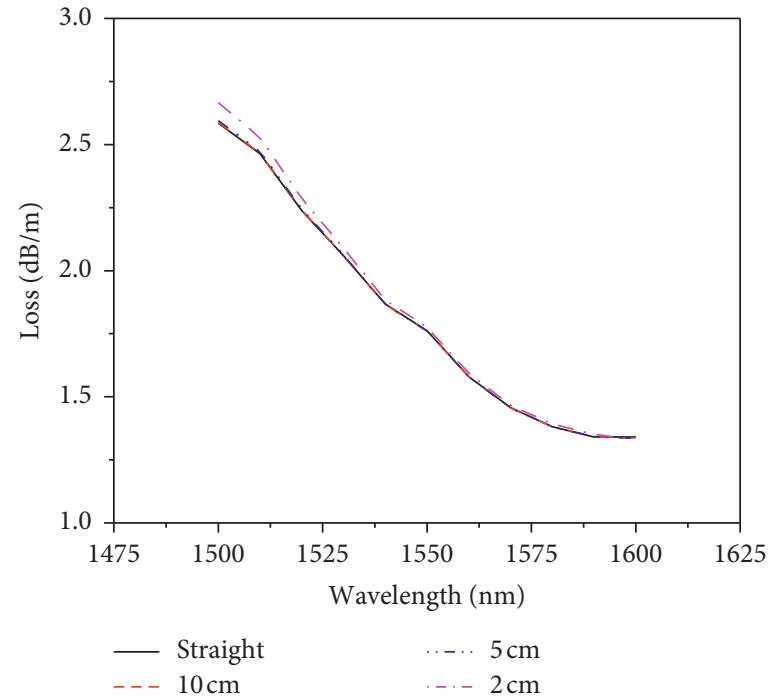

(a)

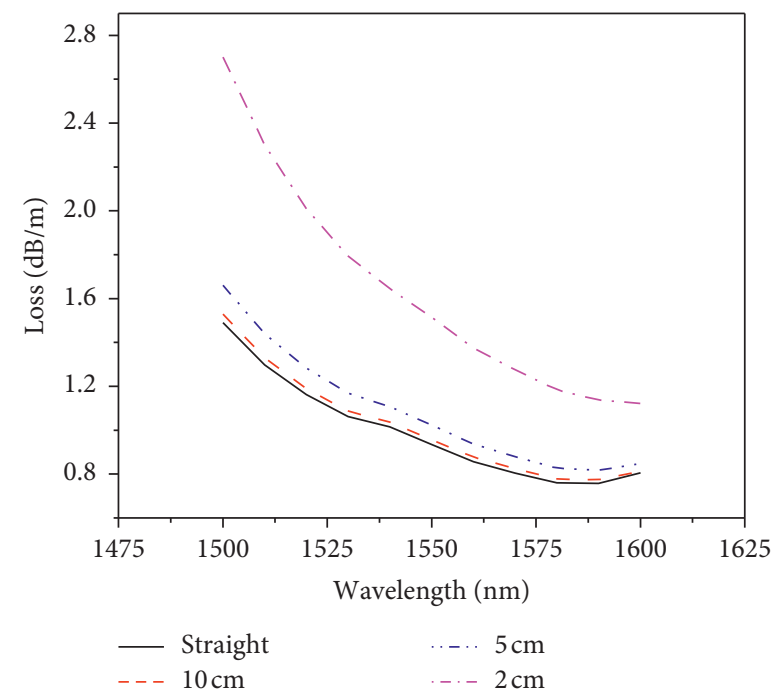

(b)

FIGURE 11: Fiber bends in $x$ direction, the bending loss of (a) $x$ polarization mode and (b) $y$ polarization mode curve at different bending radius.

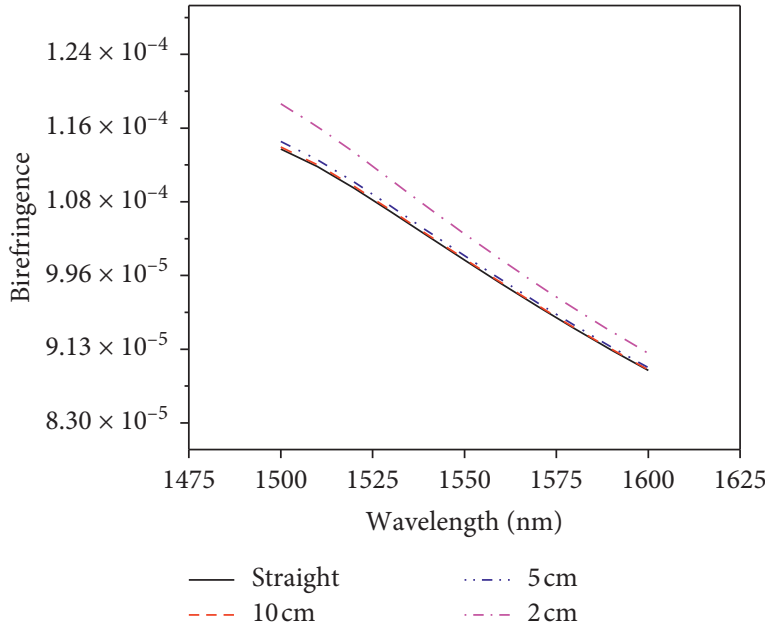

(a)

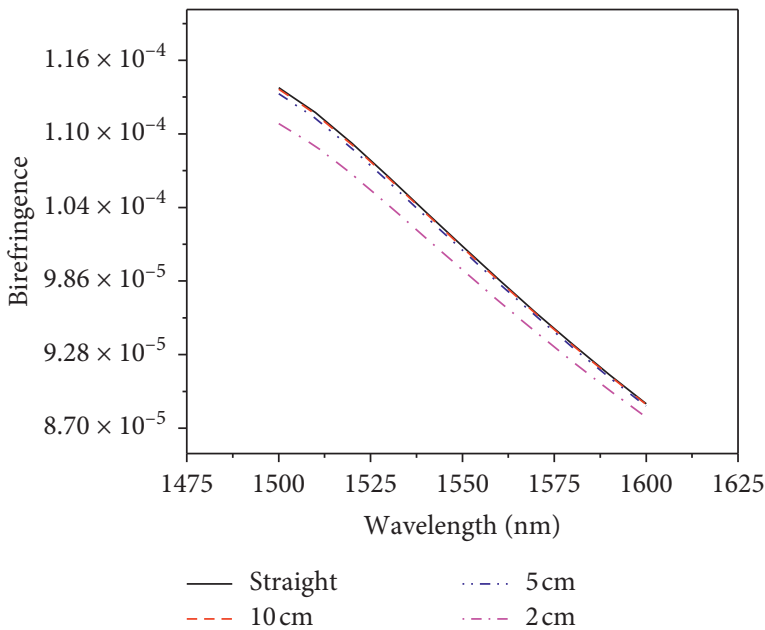

(b)

FiguRe 12: Birefringence of fiber in (a) $x$ polarization and (b) $y$ polarization as a function of wavelengths at different bending radii.

\section{Conclusions}

We propose the structure of HC-ARF with high birefringence and analyze the change of fiber loss and birefringence in the resonance region and anti-resonant region of the structure. The transmission state of the fundamental modes at the wavelengths of $1210 \mathrm{~nm}$ and $1420 \mathrm{~nm}$ in the two resonance regions in the fiber is given. These two wavelengths have been diffused into the cladding, while the fundamental mode at the wavelength of $1550 \mathrm{~nm}$ in the anti-resonant region is well bound by the optical fiber. On the other hand, through adjusting the spacing, size, and position of semicircle rings and ellipticity, we discuss the changes of birefringence, loss, and bending loss and realize the optimization of fiber structure. The simulation results indicate that the maximum birefringence is $1.2 \times 10^{-4}$ when the wavelength is $1490 \mathrm{~nm}$, and the minimum loss is $0.70 \mathrm{~dB} / \mathrm{m}$ when the wavelength is $1620 \mathrm{~nm}$. In the wavelength range of $1490 \mathrm{~nm} \sim 1550 \mathrm{~nm}$, the birefringence is more than $10^{-4}$, where the birefringence at the wavelength of $1550 \mathrm{~nm}$ is $1.01 \times 10^{-4}$, and the loss of $y$ polarization is $0.93 \mathrm{~dB} / \mathrm{m}$. If the fiber loss at $1550 \mathrm{~nm}$ is not considered, the maximum birefringence can reach $1.6 \times 10^{-4}$. Additionally, when the bending radius is more than $5 \mathrm{~cm}$, regardless of whether the fiber is bent in the $x$ direction or the $y$ direction, the bending loss does not increase significantly, indicating that it has superior bending performance. 


\section{Data Availability}

All data generated or analyzed during this study are included within the article.

\section{Conflicts of Interest}

The authors declare that there are no conflicts of interest regarding the publication of this paper.

\section{Acknowledgments}

This study was funded by the National Natural Science Foundation of China (grant nos. 61805067 and 62005076), the Science and Technology Research Project of Hebei Province Higher Education (grant no. BJ2018047), and the Natural Science Foundation of Hebei Province (grant no. F2020202069).

\section{References}

[1] Y. N. Korkishko, V. A. Fedorov, V. E. Prilutskii et al., "Strapdown inertial navigation systems based on fiber-optic gyroscopes," Gyroscopy and Navigation, vol. 5, no. 4, pp. 195-204, 2014.

[2] J. Jin, T. Zhang, L. Kong et al., "In-orbit performance evaluation of a spaceborne high precision fiber optic gyroscope," Sensors (Basel), vol. 18, no. 1, p. 106, 2018.

[3] S. Girard, J. Keurinck, Y. Ouerdane, J.-P. Meunier, and A. Boukenter, "Gamma-rays and pulsed X-ray radiation responses of germanosilicate single-mode optical fibers: influence of cladding codopants," Journal of Lightwave Technology, vol. 22, no. 8, pp. 1915-1922, 2004.

[4] C. Caillaud, C. Gilles, L. Provino et al., "Highly birefringent chalcogenide optical fiber for polarization-maintaining in the 3-85 $\mu \mathrm{m}$ mid-IR window," Optics Express, vol. 24, no. 8, pp. 7977-7986, 2016.

[5] M.-Y. Chen and Y.-K. Zhang, "Improved design of polarization-maintaining photonic crystal fibers," Optics Letters, vol. 33, no. 21, pp. 2542-2544, 2008.

[6] H. Zhuang, L. Exian, and L. Jianjun, "Hollow-core photonic quasicrystal fiber with high birefringence and ultra-low nonlinearity," Chinese Optics Letters, vol. 18, no. 3, Article ID 030603, 2020.

[7] B. Debord, A. Amsanpally, M. Chafer et al., "Ultralow transmission loss in inhibited-coupling guiding hollow fibers," Optica, vol. 4, no. 2, pp. 209-217, 2017.

[8] N. V. Wheeler, T. D. Bradley, J. R. Hayes et al., "Low loss kagome fiber in the $1 \mu \mathrm{m}$ wavelength region," in Advanced Photonics 2016 (IPR, NOMA, Sensors, Networks, SPPCom, SOF), Optical Society of America, Vancouver, Canada, 2016.

[9] M. S. Habib, O. Bang, and M. Bache, "Low-loss hollow-core silica fibers with adjacent nested anti-resonant tubes," Optics Express, vol. 23, no. 13, pp. 17394-17406, 2015.

[10] W. Belardi, "Design and properties of hollow antiresonant fibers for the visible and near infrared spectral range," Journal of Lightwave Technology, vol. 33, no. 21, pp. 4497-4503, 2015.

[11] J. R. Hayes, E. N. Fokoua, M. N. Petrovich et al., "Antiresonant hollow core fiber with an octave spanning bandwidth for short haul data communications," Journal of Lightwave Technology, vol. 35, no. 3, pp. 437-442, 2017.

[12] M. I. Hasan, N. Akhmediev, and W. Chang, "Mid-infrared supercontinuum generation in supercritical xenon-filled hollow-core negative curvature fibers," Optics Letters, vol. 41, no. 21, pp. 5122-5125, 2016.

[13] J. Sultana, M. S. Islam, C. M. B. Cordeiro et al., "Exploring low loss and single mode in antiresonant tube lattice terahertz fibers," IEEE Access, vol. 8, pp. 113309-113317, 2020.

[14] W. Lu, S. Lou, X. Wang et al., "Demonstration of low-loss flexible fiber with Zeonex tube-lattice cladding for terahertz transmission," in Proceedings of the Optical Fiber Communication Conference, Los Angeles, Ca, USA, March 2015.

[15] M. M. Nazarov, A. V. Shilov, K. A. Bzheumikhov et al., "EightCapillary cladding $\mathrm{THz}$ waveguide with low propagation losses and dispersion," IEEE Transactions on Terahertz Science and Technology, vol. 8, no. 2, pp. 183-191, 2018.

[16] A. L. S. Cruz, V. A. Serrão, C. L. Barbosa et al., "3D printed hollow core fiber with negative curvature for terahertz applications," Journal of Microwaves, Optoelectronics and Electromagnetic Applications, vol. 14, pp. SI45-SI53, 2015.

[17] J. R. Hayes, F. Poletti, M. S. Abokhamis, N. V. Wheeler, N. K. Baddela, and D. J. Richardson, "Anti-resonant hexagram hollow core fibers," Optics Express, vol. 23, no. 2, pp. 1289-1299, 2015.

[18] A. F. Kosolapov, G. K. Alagashev, A. N. Kolyadin et al., "Hollow-core revolver fibre with a double-capillary reflective cladding," Quantum Electronics, vol. 46, no. 3, pp. 267-270, 2016.

[19] L. D. Van Putten, J. Gorecki, E. Numkam Fokoua, V. Apostolopoulos, and F. Poletti, "3D-printed polymer antiresonant waveguides for short-reach terahertz applications," Applied Optics, vol. 57, no. 14, pp. 3953-3958, 2018.

[20] G. K. M. Hasanuzzaman, S. Iezekiel, C. Markos, and M. S. Habib, "Hollow-core fiber with nested anti-resonant tubes for low-loss THz guidance," Optics Communications, vol. 426, pp. 477-482, 2018.

[21] S. A. Mousavi, S. R. Sandoghchi, D. J. Richardson, and F. Poletti, "Broadband high birefringence and polarizing hollow core antiresonant fibers," Optics Express, vol. 24, no. 20, pp. 22943-22958, 2016.

[22] J. Sultana, M. S. Islam, C. M. B. Cordeiro et al., "Terahertz hollow core antiresonant fiber with metamaterial cladding," Fibers, vol. 8, no. 2, p. 14, 2020.

[23] F. Amrani, J. H. Osório, F. Delahaye et al., "Low-loss singlemode hybrid-lattice hollow-core photonic-crystal fibre," Light: Science \& Applications, vol. 10, no. 1, p. 7, 2021.

[24] N. V. Wheeler, T. D. Bradley, J. R. Hayes et al., "Low-loss Kagome hollow-core fibers operating from the near- to the mid-IR," Optics Letters, vol. 42, no. 13, pp. 2571-2574, 2017.

[25] V. Bock, M. Plötner, O. De Vries et al., "Modal content measurements $\left(\mathrm{S}^{2}\right)$ of negative curvature hollow-core photonic crystal fibers," Optics Express, vol. 25, no. 4, pp. 3006-3012, 2017.

[26] S.-F. Gao, Y.-Y. Wang, W. Ding et al., "Hollow-core conjoined-tube negative-curvature fibre with ultralow loss," Nat Commun, vol. 9, no. 1, p. 2828, 2018.

[27] T. D. Bradley, G. T. Jasion, J. R. Hayes et al., "Antiresonant hollow core fibre with $0.65 \mathrm{db} / \mathrm{km}$ attenuation across the $\mathrm{C}$ and $\mathrm{L}$ telecommunication bands," in Proceedings of the 45th European Conference on Optical Communication (ECOC 2019), Dublin, Ireland, September 2019, https://digital-library.theiet.org/content/ conferences/10.1049/cp.2019.1028. 